\title{
Chronic Kidney Disease Causes Disruption of Gastric and Small Intestinal Epithelial Tight Junction
}

\author{
Nosratola D. Vaziri Jun Yuan Sohrab Nazertehrani Zhenmin Ni Shuman Liu \\ Division of Nephrology and Hypertension, University of California, Irvine, Irvine, Calif., USA
}

\author{
Key Words \\ Gastritis · Enterocolitis · Inflammation · Malnutrition · \\ End-stage renal disease - Uremia
}

\begin{abstract}
Background: Integrity of the tight junction (TJ) which seals the gap between the epithelial cells of the gastrointestinal tract is critical in preventing the entry of the microbial toxins, antigens, and other harmful products in the subepithelial tissues and the internal milieu. By enabling the absorption of these products, impairment of the intestinal epithelial barrier leads to local and systemic inflammation. We have recently found depletion of the key protein constituents of colonic epithelial TJ in animals with chronic kidney disease (CKD). Postmortem studies have revealed the presence of inflammation throughout the gastrointestinal tract in uremic humans. This observation suggests that uremia may cause disruption of the epithelial barrier in all segments of the gastrointestinal tract including the stomach, jejunum, and ileum. The present study was undertaken to explore this possibility. Methods: Sprague-Dawley rats were randomized to CKD or control groups. The CKD group was subjected to $5 / 6$ nephrectomy while the control group underwent a sham operation. The animals were observed for 10 weeks at which time they were euthanized and their stomachs, jejunums, and ileums were removed and processed for measurement of TJ proteins. Results: The CKD rats showed marked azote-
\end{abstract}

mia, systemic oxidative stress, and marked depletion of the key protein constituents of the epithelial TJ (claudin-1, occludin, and ZO1) in the stomach, jejunum, and ileum. Conclusions: The present study extends the earlier finding of uremia-induced disruption of colonic epithelial TJ by documenting the involvement of the stomach, jejunum, and ileum as well.

Copyright $\odot 2013$ S. Karger AG, Basel

\section{Introduction}

Integrity of the gastrointestinal epithelial tight junction (TJ) which seals the gap between the epithelial cells is critical in preventing the entry of microbial toxins, antigens, and other harmful luminal contents into the submucosal tissues and eventually in the body's internal milieu. By enabling the influx of these products in the internal milieu, impairment of the intestinal epithelial barrier structure and function leads to local and systemic inflammation. Chronic kidney disease (CKD) is associated with systemic inflammation which plays a major role in the pathogenesis of cardiovascular disease, anemia, malnutrition, and various other complications [1-3]. There is mounting evidence supporting the presence of gastrointestinal barrier dysfunction and its role in the pathogenesis of systemic inflammation in uremic humans and animals [4]. For instance, intestinal permeability to large 
molecular weight polyethylene glycols is increased in uremic humans and animals $[5,6]$, luminal bacteria penetrate the intestinal wall and lodge in the mesenteric lymph nodes in uremic rats [7], hemodialysis patients commonly exhibit histological evidence of chronic inflammation throughout the gastrointestinal tract $[8,10]$, and endotoxemia is invariably present in dialysis patients in the absence of clinical infection [9-11]. These observations point to increased intestinal permeability and barrier dysfunction in patients and animals with advanced CKD. However, until recently the mechanism by which uremia increases intestinal epithelial permeability was not known. In a recent study we found heavy losses of the key protein constituents of colonic epithelial TJ in rats with CKD [12]. The depletion of intestinal epithelial TJ structure shown in that study unraveled the source of endotoxemia which is commonly present and is a major cause of inflammation in CKD $[9,11]$. In a series of subsequent in vitro studies we identified urea and the byproducts of its hydrolysis by microbial urease, i.e. ammonia and ammonium hydroxide, as the major mediator of uremia-induced disruption of the intestinal barrier function and structure [13].

As noted above, studies in uremic humans have shown evidence of local inflammation not only in the colon but throughout the gastrointestinal tract [8]. This suggests that the disruption of the epithelial barrier structure and function must occur in other segments of the gastrointestinal tract as well. The present study was undertaken to explore the effect of CKD on the epithelial TJ apparatus in the stomach, jejunum, and ileum.

\section{Methods}

\section{Animals}

Male Sprague-Dawley rats were purchased from Harlan Sprague Dawley Inc. (Indianapolis, Ind., USA). The rats were randomized to control or CKD groups. The rats assigned to the CKD group underwent 5/6 nephrectomy using dorsal incisions as described previously [8]. The control group underwent a sham operation. The animals were housed in a temperature-controlled facility with 12-hour light/dark cycles and observed for 10 weeks. Arterial blood pressure was determined by tail-cuff plethysmography as described previously [14]. At the conclusion of the observation period, the animals were placed in metabolic cages for a 24hour urine collection. They were then anesthetized (ketamine/xylazine IP) and euthanized by exsanguination using cardiac puncture. The stomach, jejunum, and ileum were removed and processed for expression of the key constituents of the TJ proteins by Western blot. All experiments were approved by the University of California, Irvine Institutional Committee for the Use and Care of Experimental Animals.
Western Blot Analyses

The tissues were homogenized on ice in modified RIPA Lysis Buffer containing $25 \mathrm{~mm}$ Tris- $\mathrm{HCl} \mathrm{pH}$ 7.4, $150 \mathrm{mM} \mathrm{NaCl}, 1 \mathrm{~mm}$ EDTA, $1 \%$ Tergitol NP-40, $0.1 \%$ sodium dodecyl sulfate, $1 \mathrm{mM}$ phenylmethylsulfonyl fluoride, and Protease Inhibitor Cocktail (Sigma-Aldrich, St. Louis, Mo., USA). Protein concentration in the tissue homogenates was determined by BSA assay kit (Pierce, Rockford, Ill., USA) and $60 \mu \mathrm{g}$ of total protein from each sample were fractionated on $4-12 \%$ Bis-Tris gradient gel (Invitrogen, Carlsbad, Calif., USA) at $120 \mathrm{~V}$ for $2 \mathrm{~h}$ and transferred to a nitrocellulose membrane. The membrane was then incubated with rabbit anticlaudin-1 or rabbit antioccludin and mouse anti-ZO1 antibodies (Invitrogen) at 1:250 dilutions and antiactin antibodies (Sigma-Aldrich) at 1:10,000 dilutions overnight. The appropriate horseradish peroxidase-conjugated secondary antibodies (SigmaAldrich) were used at a 1:5,000 dilution. The membrane was visualized with SuperSignal West Pico (Pierce) and developed by autoluminography.

Data Analysis

Student's t test was used for the statistical evaluation of the data which are presented as means \pm SD. $\mathrm{p}<0.05$ was considered significant.

\section{Results}

\section{General Data}

Compared to the control group, the CKD rats exhibited significant reductions in body weight ( $503 \pm 25$ vs. $462 \pm 18 \mathrm{~g}, \mathrm{p}<0.01)$ and hematocrit $(45 \pm 1.2$ vs. $37 \pm$ $1.4 \%, \mathrm{p}<0.01$ ), and significant increases in systolic arterial pressure $(121 \pm 3.4$ vs. $155 \pm 2.8 \mathrm{~mm} \mathrm{Hg}, \mathrm{p}<0.01)$, urine protein excretion $(10.2 \pm 2.5 \mathrm{vs} .143 .5 \pm 9.7 \mathrm{mg} / 24 \mathrm{~h}$, $\mathrm{p}<0.01)$, plasma creatinine concentration $(0.4 \pm 0.1$ vs. $1.4 \pm 0.2 \mathrm{mg} / \mathrm{dl}, \mathrm{p}<0.01)$, and urea ( $45 \pm 5.1$ vs. $98 \pm 6.4$ $\mathrm{mg} / \mathrm{dl}, \mathrm{p}<0.01)$ concentration. This was associated with a marked increase in plasma malondialdehyde level $(0.8 \pm$ 0.1 vs. $1.5 \pm 0.2 \mathrm{umol} / \mathrm{ml}$ ) denoting the presence of systemic oxidative stress.

\section{The TJ Protein Data}

Data are illustrated in figures 1-3. Compared with the control group the untreated CKD group showed a marked reduction in protein abundance of the key transcellular TJ proteins, claudin- 1 and occludin in the gastric tissue. The reduction in the gastric tissue claudin- 1 and occludin abundance in the CKD rats was accompanied by a marked reduction of the main cytosolic plaque TJ protein, ZO1. Likewise the claudin-1, occludin, and $\mathrm{ZO} 1$ abundance in the jejunum and ileum was markedly lower in the CKD animals than the corresponding values found in the control group. 


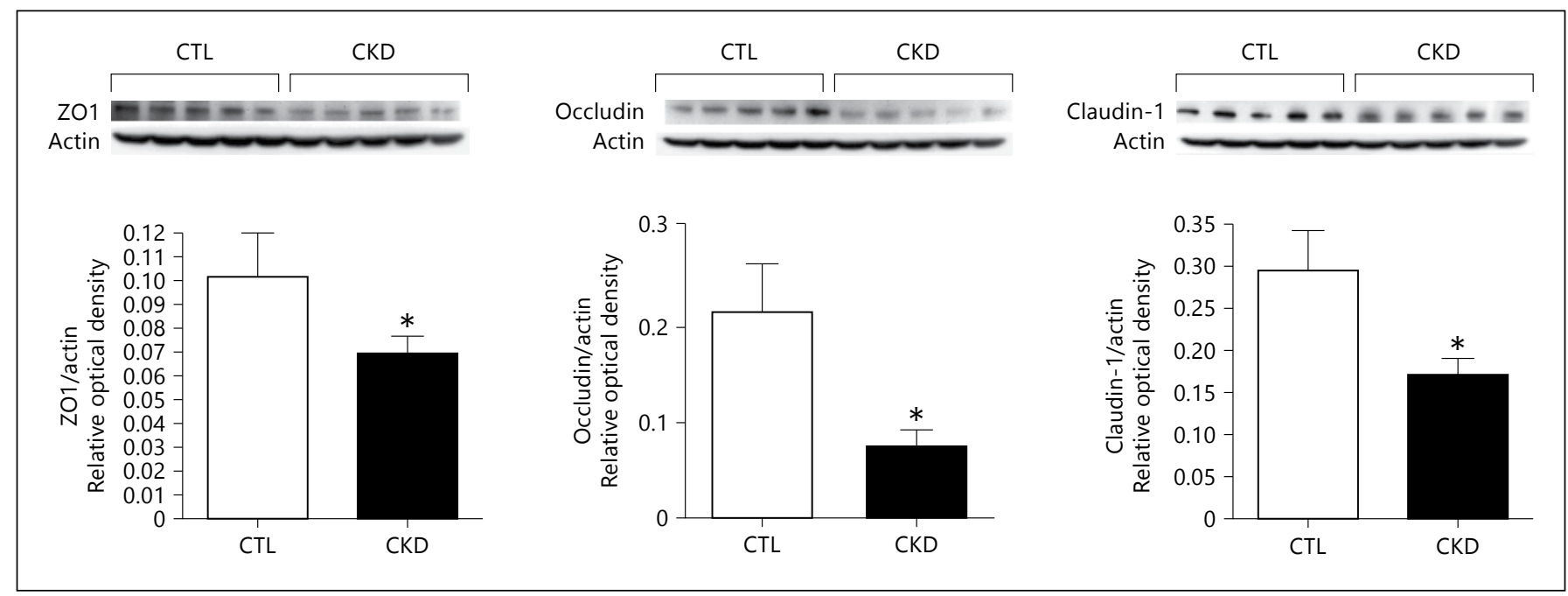

Fig. 1. Representative Western blot and group data depicting protein abundance of ZO1, claudin-1, and occludin in the stomach of normal control (CTL) and CKD rats. ${ }^{*} \mathrm{p}<0.05$.

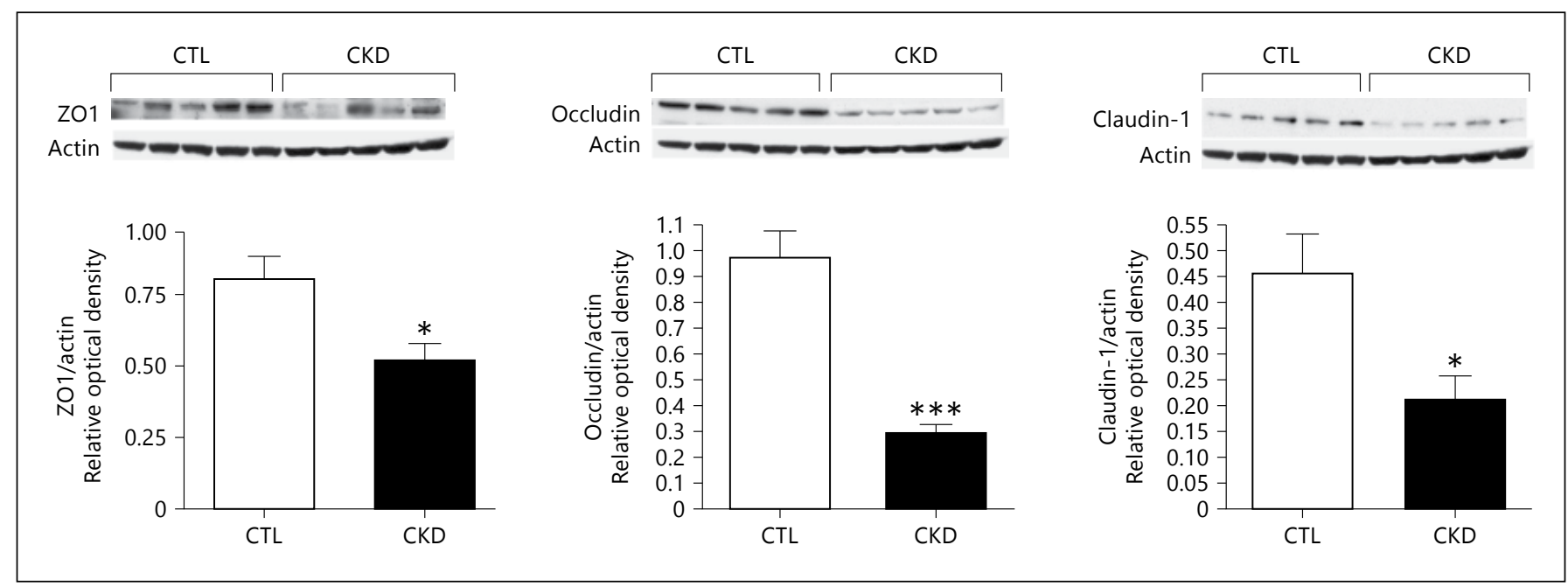

Fig. 2. Representative Western blot and group data depicting protein abundance of ZO1, claudin-1, and occludin in the jejunum of normal control (CTL) and CKD rats. ${ }^{*} \mathrm{p}<0.02$; $^{* * *} \mathrm{p}<0.001$.

\section{Discussion}

The central finding of the present study is the demonstration of a significant reduction in the key protein constituents of the epithelial TJ in the stomach, jejunum, and ileum of animals with CKD as compared to those found in the normal control group. These findings extend the results of our earlier studies which showed disruption of the colonic epithelial TJ in two different models of CKD induced by either subtotal nephrectomy or consumption of an adenine-containing diet which leads to intense chronic interstitial nephropathy [12].

The TJ apparatus consists of the transmembrane, cytosolic, and perijunctional proteins. The transmembrane proteins include the occludin and claudin protein families which form the barrier against influxes of fluids, solutes, and particulate matters by connecting the plasma membranes of the adjacent cells. The cytosolic proteins, 


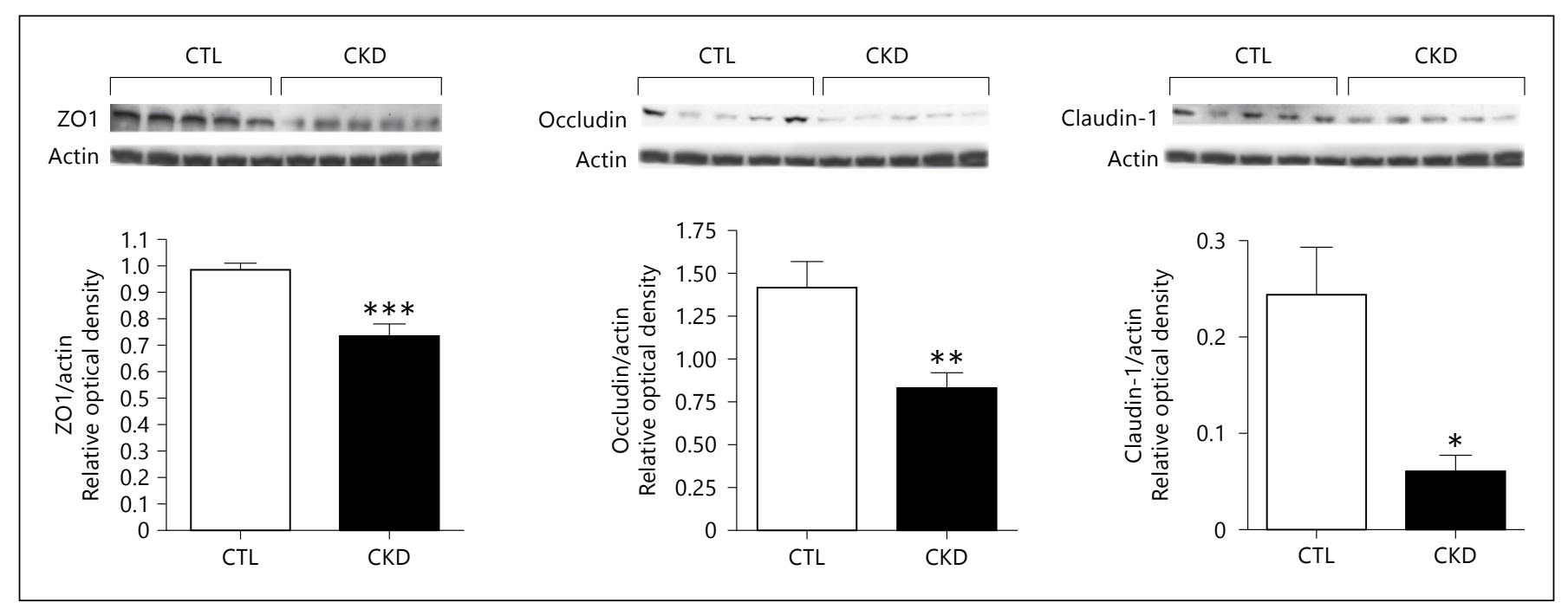

Fig. 3. Western blot and group data depicting protein abundance of ZO1, claudin-1, and occludin in the ileum of normal control (CTL) and CKD rats. ${ }^{*} \mathrm{p}=0.02{ }^{* *} \mathrm{p}=0.01{ }^{* * *} \mathrm{p}=0.001$.

i.e. the zonula occludens ( $\mathrm{ZO})$ protein family, form an anchor which simultaneously binds the intracellular domains of occludin and claudin and the perijunctional actin-myosine ring $[15,16]$. The integrity of the TJ is essential for preservation of the epithelial barrier function. Consequently the CKD-induced depletion of the key TJ protein constituents in the colon shown in our earlier studies [12] and in the stomach, jejunum, and ileum found in the present study contributes to the associated local and systemic inflammation.

Using cultured human colonocytes, we recently found a marked reduction in the transepithelial electrical resistance and depletion of the TJ proteins following incubation in culture media containing prehemodialysis plasma from patients with advanced CKD [17]. The epithelial barrier-damaging effects of the uremic plasma were significantly less intense when cells were exposed to the posthemodialysis plasma from the same patients. In contrast, plasma from healthy control persons did not significantly alter either the transepithelial electrical resistance or the abundance of TJ protein. These observations pointed to role of a dialyzable uremic retention product in promoting disruption of the intestinal epithelial barrier structure and function. In a subsequent study, we identified urea and the byproducts of its hydrolysis by urease, i.e. ammonia and ammonium hydroxide, as the major cause of disruption of the colonic epithelial TJ [13]. Accumulation of urea in the body fluids in humans and animals with renal failure leads to its heavy influx into the gastrointestinal tract via diffusion and incorporation in glandular secretions [18]. Within the intestinal lumen, urea is hydrolyzed by microbial urease to form large quantities of ammonia $\left[\mathrm{CO}\left(\mathrm{NH}_{2}\right) 2+\mathrm{H}_{2} \mathrm{O} \rightarrow \mathrm{CO}_{2}+\right.$ $2 \mathrm{NH}_{3}$ ] which is readily converted to ammonium hydroxide $\left[\mathrm{NH}_{3}+\mathrm{H}_{2} \mathrm{O} \rightarrow \mathrm{NH}_{4} \mathrm{OH}\right][19,20]$. Ammonium hydroxide can, in turn, interact with and dissociate the TJ proteins which directly face the lumen and are exposed to the luminal contents. Uremia-induced diffusion of urea occurs uniformly throughout the length of the gastrointestinal tract via incorporation in the glandular secretions including saliva, gastric juices, bile, pancreatic secretions, and products of mucosal glands. The heavy influx of urea in the gastrointestinal tract is compounded by microbial colonization of the upper intestinal tract and dramatic change in the composition of the gut microbiome in the uremic humans and animals [21]. It is therefore not surprising that uremia leads to the epithelial TJ disruption and barrier dysfunction throughout the length of the gastrointestinal tract. Given the role of urea and urea-derived ammonia in the pathogenesis of CKDassociated disruption of the intestinal barrier structure, in a recent study we explored the effect of oral activated charcoal adsorbent [22] on colonic epithelial TJ in uremic animals. The study revealed significant improvement in the epithelial TJ in the oral adsorbent-treated CKD rats. This was associated with significant attenuation of the systemic oxidative stress, inflammation, and endotoxemia. 
In conclusion, the present study demonstrated marked disruption of the gastric, jejunal, and ileal epithelial TJ in uremia. These findings extend the results of our earlier studies which showed depletion of the colonic epithelial $\mathrm{TJ}$ in the animal models of CKD and in cultured human colonocytes in vitro.

\section{Disclosure Statement}

The authors have no conflict of interest to declare.

\section{References}

1 Cachofeiro V, Goicochea M, de Vinuesa SG, Oubiña P, Lahera V, Luño J: Oxidative stress and inflammation, a link between chronic kidney disease and cardiovascular disease. Kidney Int Suppl 2008;111:S4-S9.

2 Stenvinkel P: Inflammation in end-stage renal disease: the hidden enemy. Nephrology (Carlton) 2006;11:36-41.

3 Ruiz S, Pergola PE, Zager RA, Vaziri ND: Targeting Nrf2 activation to ameliorate oxidative stress and inflammation in chronic kidney disease. Kidney Int 2013;83:1029-1041.

$\checkmark 4$ Vaziri ND: CKD impairs barrier function and alters microbial flora of the intestine - a major link to inflammation and uremic toxicity. Curr Opin Nephrol Hypertens 2012;21:587592.

5 Magnusson M, Magnusson KE, Sundqvist T, et al: Increased intestinal permeability to differently sized polyethylene glycols in uremic rats: effects of low- and high protein diets. Nephron 1990;56:306-311.

6 Magnusson M, Magnusson KE, Sundqvist T, et al: Impaired intestinal barrier function measured by differently sized polyethylene glycols in patients with chronic renal failure. Gut 1991;32:754-759.

$\checkmark 7$ de Almeida Duarte JB, de Aguilar-Nascimento JE, Nascimento $M$, et al: Bacterial translocation in experimental uremia. Urol Res 2004; 32:266-270

8 Vaziri ND, Dure-Smith B, Miller R, et al: $\mathrm{Pa}$ thology of gastrointestinal tract in chronic hemodialysis patients: an autopsy study of 78 cases. Am J Gastroenterol 1985;80:608-611. $\checkmark 9$ Feroze U, Kalantar-Zadeh K, Sterling KA, Molnar MZ, Noori N, Benner D, Shah V, Dwivedi R, Becker K, Kovesdy CP, Raj DS: Examining associations of circulating endotoxin with nutritional status, inflammation, and mortality in hemodialysis patients. J Ren Nutr 2012;22:317-326.

10 Gonçalves S, Pecoits-Filho R, Perreto S, et al: Associations between renal function, volume status and endotoxaemia in chronic kidney disease patients. Nephrol Dial Transplant 2006;21:2788-2794.

11 Szeto CC, Kwan BC, Chow KM, et al: Endotoxemia is related to systemic inflammation and atherosclerosis in peritoneal dialysis patients. Clin J Am Soc Nephrol 2008;3:431436.

12 Vaziri ND, Yuan J, Rahimi A, Ni Z, Said H, Subramanian VS: Disintegration of colonic epithelial tight junction in uremia: a likely cause of CKD-associated inflammation. Nephrol Dial Transplant 2012;27:2686-2693.

13 Vaziri ND, Yuan J, Norris K: Role of urea in intestinal barrier dysfunction and disruption of epithelial tight junction in chronic kidney disease. Am J Nephrol 2013;37:1-6.

14 Vaziri ND, Ni Z, Oveisi F, et al: Effect of antioxidant therapy on blood pressure and $\mathrm{NO}$ synthase expression in hypertensive rats. Hypertension 2000;36:957-964
15 Turner JR: Intestinal mucosal barrier function in health and disease. Nat Rev Immunol 2009;9:799-809.

16 Nusrat A, Turner JR, Madara JL: Molecular physiology and pathophysiology of tight junctions. IV. Regulation of tight junctions by extracellular stimuli: nutrients, cytokines, and immune cells. Am J Physiol Gastrointest Liver Physiol 2000;279:G851-G857.

17 Vaziri ND, Goshtasbi N, Yuan J, Jellbauer S, Moradi H, Raffatellu M, Kalantar-Zadeh K: Uremic human plasma degrades intestinal epithelial barrier structure and function. Am J Nephrology 2012;36:438-443.

18 Lee YT: Urea concentration in intestinal fluids in normal and uremic dogs. J Surg Oncol 1971;3:163-168.

19 Bourke E, Milne MD, Stokes GS: Caecal pH and ammonia in experimental uraemia. Gut 1966;7:558-561.

20 Swales JD, Tange JD, Evans DJ: Intestinal ammonia in uraemia: the effect of a urease inhibitor, acetohydroxamic acid. Clin Sci 1972 42:105-112.

21 Vaziri ND, Wong J, Pahl MV, Piceno YM, Yuan J, De Santis TZ, Ni Z, Nguyen TH, Andersen GL: Chronic kidney disease alters the composition of intestinal microbial flora. Kidney Int 2013;83:308-315.

22 Vaziri ND, Yuan J, Khazaeli M, Masuda Y, Ichii H, Liu S: Oral activated charcoal adsorbent (AST-120) ameliorates CKD-induced intestinal epithelial barrier disruption and systemic inflammation. Am J Nephrol 2013; 37:518-525. 